5 Köpfe von Nebelkrähen. Corvus cornix mit Schnabelmifsbildungen. Ulmenhorst.

1 Buchfink. Fringilla coeleps juv. Ulmenhorst.

1 Birkenzeisig. Acanthis linaria. Ulmenhorst.

1 Rohrammer. Emberiza schoeniclus, Form microrhynchus @. Ulmenhorst.

1 Feldlerche. Alauda arvensis ơ. Ulmenhorst.

1 Heckenbraunelle. Accentor modularis mit Ring No 1432. Lübeck.

1 Wachbolderdrossel. Turdus pilaris o. Ulmenhorst.

1 Amsel. Turdus merula 우 mit Ring Nr. 1723. Lübeck.

22 Vögel.

\title{
Über den Vogelzug 1911 bei Lübeck.
}

\section{Von Werner Hagen.}

Die Zugsforschung ist aufser der Ethologie noch immer das schwierigste Kapitel der Ornithologie. Es herrscht auf diesem Gebiete jedoch so kernfrisches Leben, dafs man sich den schönsten Hoffnungen für die Zukunft hingeben kann: Eine Reihe von „Ornithologischen Zentralen", mehrere „Vogelwarten" arbeiten an diesem Problem. Die Zahl der letzteren ist jedoch noch zu spärlich, auch für Deutschland.

Durch die Ringexperimente ist zwar festgestellt, dafs viele Vögel ,für sich" bummeln. Aber Tatsache bleibt, dafs an manchen Tagen, besonders aber in Nächten gröfsere Vogelmassen gemeinsam, wenn auch von einander unabhängig, in Bewegung sind. Nun treten jedoch diese nächtlichen Züge, die das sicherste und bequemste Vergleichsmaterial bilden, bei der einzigen Ostseestation, Rossitten, nicht in Erscheinung. Die Gründe möchte ich weiter unten ausführlich behandeln. Tageszug, gefälscht durch Rast, ist aber sehr schwer zu vergleichen. Wie sich aufserdem aus der Literatur ersehen läfst, stehen den nordischen Vögeln in unserm Ostseegebıet 3 „Einfallstore“ zur Verfügung: Kurische Nehrung, Odergebiet, Trave. Bei Rossitten sind die Verhältnisse anscheinend am kompliziertesten. Die von der finnisch-russischen Küste und dem Seengebiet kommenden Vögel ziehen teils südlich, teils südwestlich ins Binnenland, teils folgen sie westlich der Küste. Die an der schwedischen Küste entlangziehenden Vögel gehen über Kügen oderaufwärts. Woher nun die grofsen Zugsinassen stammen, die, von der mecklenburgischen Küste kommend, in grofsen Scharen durch die Trave, die ihnen den Weg ins Binnenland öffnet, fluten, läfst sich schwer angeben. Gewifs wird der lübecksche Zug mit dem Rossittener in Verbindung stehen. Jedoch scheint der Zusammenhang sehr locker und nur für manche Strandvögel giltig zu sein. Das Frühjahr 
1909 stellte fest, dafs Lübeck mit Rossitten nichts gemein hatte. Ich vermutete daher ursprünglich, dafs der hiesige Zug in besonderem Verhältnis zu dem Helgoländer stände. Doch ergab ein Vergleich des Materials von 1909 mit grofser Bestimmtheit, dafs beide unabhängig von einander sind. Auch Herr Dr Weigold kommt zum gleichen Resultat. „Daraus (den nächtlichen Zügen, Hg.) ergibt sich mir mit voller Sicherheit, dafs in der Regel Ihr Zug mit dem Helgoländer nichts direkt zu tun hat." Als Beweis gestattete mir Herr Dr. W., seine Helgoländer Randbemerkungen zum Vergleich aufzuführen. Dafs auch der Lübecker Zug mit dem der Oderstrafse nicht in eigentlicher Beziehung steht, ergibt der Zug des Grofsen und Regenbrachers im Ostseegebiet. Beide erscheinen im Westwinkel desselben weit früher als im Odergebiet. Wüstnei vermutet zwar in seiner mir erst kürzlich zu Händen gekommenen Arbeit: Der Vogelzug in Mecklenburg, J. f. 0. 1902, p. 238-258, 266-278, dafs diese westmecklenburgischen Küstenwanderer vom Darfs und von Rügen stammen, gibt aber keinen Beweis, sondern schreibt vorsichtshalber, dafs diese Vögel ,a $\mathrm{uch}$ wohl die nach SW verlaufende Küste verfolgen". Dagegen weist er nach, was mir sehr interessant war, dafs die Vögel, die das Innere Mecklenburgs südwestlich durchziehen und an den Landseen des Ostens rasten, vom Darfs und von Rügen stammen, dafs aber die westmecklenburgischen Küstenwanderer mit den Seen, selsbt mit dem Schweriner See, nicht in Beziehungen stehen. Ich hatte darüber erst eine Beobachtung. „Nördlich von Wismar gelangt der grofse Strom der Strandvögel aus NO über die Halbinsel Wustrow nach dem langen Werder und der Nordküste von Poel, streift diese und setzt von hier über die Wismarsche Bucbt nach dem Tarnewitzer Ort, der Nordwestecke des Wohlenberger Wieks über. Von hier geht der Hauptzug weiter nach W an der Küste entlang." Wüstnei's Beobachtungen stimmen Punkt für Punkt mit den meinen überein, nur seine Zugszeiten weisen einige Fehler auf. Ich konnte nachweisen (J. f. O. 1910), dafs jener Zug der Küste bis zur Klützer Ecke folgt, dann südwestlich in die Lübecker Bucht biegt und direkt auf die Travemündung zusteuert. Hier wird er durch einen schwachen Zug verstärkt, der von der ostholsteinischen Küste kommt. Die Trave führt den Strom der Wanderer südwestlich ins Binnenland. Bevor nicht sicheres Material vorliegt, bin ich der Ansicht, dafs die Lübecker Wanderer von Westschweden über die dänischen Inseln ziehen und dann auf die mecklenburgische Küste stofsen, die sie zur Trave führt, welche ihre „Einfallspforte“ ins Binnenland bildet (siehe Vögel Lübecks). Auch Wüstnei (a. a. 0. p. 241) schreibt: ,Ferner kommen auch über die dänischen Inseln nordische Wanderer an unsere Küste“. Lübecks Vogelzug ist demnach ein ,eigener", der nur wenig mit den beiden andern Ostseezügen in Verbindung steht. 
Es genügt also die eine Warte an der Ostsee nicht, sondern es wäre wünschenswert, dafs im Odergebiet und an der Trave noch je eine hinzukäme, wenn planmärsig der gesamte Zug in seinen Hauptphasen bearbeitet werden soll.

\section{Wann und weshalb rufen die nächtlichen Wanderer?}

Das bequemste und sicherste Vergleichsmaterial in der Zugsforschung bieten die nächtlichen Züge. Sie sind in der Regel stärker als die Tageszüge, geben dem Vogel keine Gelegenheit, vor der Morgendämmerung einzufallen, werden durch Rast nicht gefälscht. Dem Auge werden sie nur da bemerkbar, wo starke Lichtscheine teilweise sie in ihren Bereich kommen lassen. Also ist es das $\mathrm{Ohr}$, das sie am sichersten fixiert. Denn auf ihren Wanderungen lassen die Vögel Rufe hören.

Prof. Dr. Häcker schreibt in: Der Gesang der Vögel, seine anatomischen und biologischen Grundlagen, Jena 1900, p. 39: „Von dem ursprünglichen Lockruf . . . . . würde sich der Signalruf darin unterscheiden, dafs er von den gesellig lebenden Vögeln auch dann, wenn kein Mitglied der Gesellschaft fehlt, ohne dafs also eine besondere Erregung ${ }^{1}$ ), es sei denn die mit der physischen Anstrengung verbundene, vorliegt, im Flug und überhaupt während der Bewegung fast ununterbrochen ${ }^{1}$ ) ausgestofsen wird. Der Signalruf stellt also einen gewissermafsen zur Gewohnheit gewordenen ${ }^{1}$ ) Lockruf dar, und zwar dient er den Vögeln als fortwährendes Signal ${ }^{1}$ ), durch welches sie unbewufst zum beständigen Zusammenschlufs veranlafst werden, ebenso wie die Glocken des Weideviehs den Zusammenhalt der Herde zum Zweck haben.“ p. 40: „Eine ganz hervorragende Bedeutung gewinnt der Signalruf beim Wandern der Zugvögel Bei diesen mit gröfster Geschwindigkeit (?!) sich vollziehenden Reisen wird von den meisten Arten fast ununterbrochen gelockt, und man kann sich leicht denken, dafs gerade bei ungünstigen Witterungsverhältnissen, namentlich in stürmischen, finsteren Nächten, dieses Rufen und Locken in ähnlicher Weise unentbehrlich ist, wie in nebelreichem Fahrwasser die Signale des Nebelhorns und der Glockenbojen. Welche Bedeutung diese Signalrufe für das Zusammenhalten der Artgenossen haben, darüber gewinnt man ein besonders anschauliches Bild, wenn man die Beschreibung liest, welche Gätke von einem der nächtlichen Massenzüge gegeben hat." (Folgt eine Schilderung aus: Gätke, die Vogelwarte Helgoland.)

Es ist nun im letzten Jahrzehnt das Interesse für die Vogelwissenschaft, damit auch für die Zugsforschung, bedeutend gestiegen. Der Kreis der Ornithologen hat sich vergröfsert. Auch

1) Vom Autor selbst gesperrt. 
Jagdschriften richten dem Vogelzuge eine Extraspalte ein und geben am Schlufs der Zugzeit eine Zusammenfassung ihresMaterials. Durchblättert man jedoch die Literatur, so findet man über nächtliche Züge nur ganz dürftige Angaben, die stets von e i n z eln e n Leuchttürmen oder gröfseren Städten stammen. Da heute aber auch auf dem Lande genügend Vogelkenner wohnen, auch schriftstellernde, da bei Rossitten keine Nachtzüge festgestellt werden, trotz der bedeutenden Zugstrafse, da ferner Dr. Weigolds Aufruf in der Marinerundschau um Angabe nächtlicher Vogelzüge auf See bisher vergeblich war, da aufserdem befreundete Schiffer der Ostsee mir Mitteilungen von ermattet aufgeflogenen Wanderern, niemals aber von nächltichen Vogelrufen auf See machen konnten, so kann die allgemeine, auch von Häcker geteilte Annahme, dafs die nächtlichen Wanderer ununterbrochen locken, nicht richtig sein. Wo bleiben die Riesenschwärme von Helgoland, wo die Massenzüge von Lübeck, weshalb spürt Rossitten nichts vom Nachtzuge? Das sind Fragen, die unlöslich scheinen, da allein durch die Rufe die Schwärme ihren Verbleib nachweisen. Dr. Thienemann schrieb einmal, man müsse den Vogelzügen im Luftschiff folgen. Bevor aber das zu verwirklichen ist, mufs man die Lösung in anderer Weise aufzufinden suchen. Bei Rossitten ist an eine "Verfolgung" der Nachtwanderer wegen des anscheinenden Fehlens derselben und wegen der Geländeschwierigkeiten unausführbar, bei Helgoland desgleichen, da dem Wart ein Extra-Dampfer zur Verfügung stehen müfste, was wohl zu kostspielig sein wird. Bei Lübeck ist sie jedoch möglich, da der nordost-südwest verlaufende Zug die Richtung Lübeck-HamburgBremen direkt innehält. Diese Orte sind durch gute Chausseen verbunden. Mit einem Auto könnten die Züge leicht begleitet werden. Wir würden dann den Verbleib der Lübecker Wanderer auf eine weite Strecke feststellen können, wenn - die Vögel ununterbrochen rufen täten. Wie ich oben nachwies, ist das auf Grund der fehlenden Literatur $\mathrm{zu}$ bezweifeln. Ich achtete in letzten Jahren besonders auf diese Frage, da sie sich in Deutschland nirgends besser lösen läfst als bei Lübeck. Über diese Stadt geht ein starker Zug, über ihr rufen die Vögel sehr lebhaft. Es lag mir daran, festzustellen, ob das auch aufserhalb des Lichtkreises der Stadt der Fall sei. Ich habe nachts viel gestreift. Selbst im Travegebiet habe ich nachts selten Rufe der Wanderer vernommen. Nur von da ab, wo bei ca. $2-300 \mathrm{~m}$ die Lichtzone der Stadt beginnt, nur von dort bis über die Stadt vernahm ich die Laute der Zügler, der nordost-südwest, resp. umgekehrt Vorübereilenden. Die Stadt hat öfters auf die Ziehenden Einflufs. Im Frühling im N., im Herbst im S. von Lübeck hört man Schwärme, die zum Lichtkreis zurückkehren und über der Stadt kreisen, bis sie den richtigen „Anschlufs" gefunden haben. Im 0 . von $\mathrm{I}_{\text {. }}$ hörte ich in Zugnächten aufserhalb der Lichtzone nur spärliche Laute, je näher ich der Stadt, also dem Lichtkreis 
kam, desto lauter und häufiger erschallten sie. Sichere Gewilsheit bekam ich in diesem Jahre (1911). Als in der Nacht vom 1. zum 2. April der stärkste der von mir in dem Frühling beobachteten Züge stattfand, begab ich mich (ich wohne am Nordrand Lübecks) in die Felder nördlich von Lübeck, um ganz ohne Strafsenlärm den Zug ungestört beobachten zu können. Schon gleich fiel mir auf, dafs der Lärm über der Innenstadt viel gröfser als hier vor dem Tore sei. Als ich aber das nur 5 Min. entfernte Feld, damit die Dunkelheit, betrat, herrschte über mir fast vollständige Stille. $\mathrm{Ab}$ und $\mathrm{zu}$ nur liefs sich eine Sing-oder Weindrossel oder Bachstelze hören, ganz selten flüchtig ein Brachvogel-, Möwen- oder Bekassinenruf. Ich ging daher zur Israelsdorfer Allee und hatte über den Laternen sofort wieder lebhafte Rufe. Ununterbrochen aber schrie es fern über der Stadt. Ich ging bis Karlshof. Nachdem die letzte Laterne passiert war, kaum noch ein Laut. Im Israelsdorfer Forst vollständige Stille. Nur wenn ein riesiger Lachmöwen- oder Bracherschwarm von der Stadt kam, klangen die Rufe noch eine Weile, um bis zu mir immermehr abzusterben. Weiter nach NO, zur See hin, war es vollständig still. Über der Stadt rief es fortwährend. Jch ging zurück. Sowie ich die Laternen wieder erreicht hatte, scholl über mir ein Geschrei, dafs zur Stadt hin ständig zunahm. Ich setzte mich im Stadtpark hin und hatte nun vor mir über der inneren Stadt die ununterbrochenen Rufe, über mir die abnehmenden, pausierenden, ganz hinter mir die Stille.

Nach den Beobachtungen dieser bedeutenden Zugnacht war es mir klar, dafs Häckers Ansicht von der Bedeutung der Wanderrufe vollständig irrig ist.

Im Lichtkreis der Stadt, des Leuchtturmes werden die Rufe erschallen lassen, in der Dunkelheit verstummen sie. Sie werden also $\mathrm{n}$ i c h t ununterbrochen ausgestofsen. Sie sind also ke in e Signale zum Zusammenhalten. Am Tage freilich rufen dieselben Vögel fast ununterbrochen. Aber dann liegen ganz andere Verhältnisse vor. Sie haben bei ihrem Überflug dann das Bild der Erde unter sich und sehen ihre Feinde. Man braucht sich nur mit der Flinte an den Strand zu stellen, um zu hören, wie das Geschrei der Bracher ${ }^{1}$ ) lebhafter wird. Die grofse Menschenmenge am Badestrand veranlafst sie ebenfalls, lauter zu rufen. Es sind also keine Signale zum Zusammenhalten, sondern lediglich Warnrufe oder gegenseitige Ermunterungen zur Vorsicht. Wenn es nur Signale zum Zusammenhalten wären, dann müfsten kleine Scharen von 2 bis 3 Exemplaren, die sich also immer sehen können, schweigen. Man kann hier bei L. aber leicht die Beobachtung machen, dafs am Tage kleine Bracherschwärme im Verhältnis mehr rufen als gröfsere. Die Vögel fühlen sich eben im grofsen Schwarm sicherer. Die Vögel eines grofsen Schwarmes schweigen weite

1) Ich nehme diesen Vogel als Beispiel, weil es ein sehr typisches ist. 
Strecken weit vollständig, nur wenn ein Mensch, ein Fischerboot unter ihnen ihre Strafse kreuzt, dann schreien die meisten los, um nachher wieder still zu werden; die Vögel kleiner Schwärme rufen weit häufiger. Es sind das Beobachtungen, die ich oft machte. Selbst einzeln ziehende Bracher, bei denen die Laute nach Häckers Ansicht durchaus unnötig wären, rufen unausgesetzt. Sie fühlen sich ungemütlich und ängstlich. Deshalb sind sie durch Anpfeifen am leichtesten zu übertölpeln, weil sie bei den vermuteten Artgenossen Anschlufs suchen. Die Angabe, dafs nur die Gewohnheit die Rufe veranlafst, ist zu billig, um glaubhaft zu erscheinen. Die Wanderrufe sind also keine Signale zum Zusammenhalten, die ohne besondere Erregung ausgestofsen werden, sondern sie werden ausgestofsen, weil ein e psych is che Erregung vorliegt. Fast jede Erregung findet ja beim Vogel im Rufe Auslösung. Man kann diese Rufe am treffendsten mit Angst- oder Warnrufen bezeichnen.

Nach dieser Auffassung ist es leicht erklärlich, weshalb die Vögel nachts nur im Lichtkreis der Stadt und der Leuchttürme rufen. Wenn sie nachts über die Erde fliegen, sind ihnen die Einzelheiten der Oberfläche verhüllt, vielleicht nur schattenhaft heben sich ihnen Wälder, Flüsse usw. ab. Ein Grund zur Beunruhigung, zum Rufen also, liegt nicht vor. Da taucht plötzlich eine grofse Helligkeit wie Riesenfeuer oder wie Glühwurmsleuchten auf. Sie sind von einem Lichtmeer umgeben oder getroffen vom blendenden Strahl. Sie geraten in Aufregung und - rufen. Sind es nun Riesenschwärme, aufgestaut durch widrige meteorologische Verhältnisse, so läuft dem Helgoländer über die fliegende Fleischmenge das Wasser im Munde zusammen, der Lübecker flucht über den entbehrten Nachtschlaf.

Würde Häckers Ansicht richtig sein, so mülsten gerade dann die Vögel schweigen, da sie sich gegenseitig ja sehen können, und da jedes Stück die Richtung der Schwärme sieht. (Über Lübeck ziehen in manchen Nächten die Vögel sichtbar.) Die Dunkelheit mürste sie wieder zum Rufen veranlassen. Die Tatsachen sind aber direkt entgegengesetzt. Daher ist es leicht erklärlich, weshalb Nachrichten über nächtliche Züge nur von einzelnen Leuchttürmen und Städten vorliegen.

Es ist daher aber auch leicht einzusehen, weshalb der Aufruf von Dr. Weigold resultatlos blieb, weshalb bei Rossitten trotz der grofsen Zugs- und Raststation keine Nachtzüge festzustellen sind. Über den Verbleib der Lübecker Züge läfst sich einiges vermuten. Die Richtung weist auf Hamburg. Als im Herbst 1910 bei Lübeck die Singdrosseln zu ziehen begannen, hörte ich auch über Hamburg nachts $\mathrm{NO}-\mathrm{SW}$-lich Ziehende. Ein Bekannter erzählte, dafs er nachts über Hamburg „Regenpfeifer" gehört hätte. Die Richtung weist weiter auf Westfalen: Aus Münster liegen Angaben in der Literatur über nächtliche Bracherzüge vor. Bekannte Ornithologen in Bielefeld und Capelle 
konnten mir Nachrichten tber nächtliche Zäge dieser Art in NOSW-licher Richtong geben.

Oft liest man. dsls in Mondnächten kein Zug stattfinde. Auch das ist irrig. Ich babe in manchen Mondoächten Züge feststellen konnen. Allerdings hört msn bei Mondschein, wie tiberhaupt in bellen Nächten, sehr selten Vogelrufe. Sie klingen in solchen Nächten riel leiser. Die Vögel riehen dann anscheinend bober, da die Erdobertische innen leichter erkenubar sein wird. Weil sie darum in den hellen Nächten rom Leachtturm, ron den Stidten weniger alteriert werden, rufen sie wenig oder gar nicht und werden dann nicht fxiert. Hullt sich die Brdobertäche sber in Nebel, so sind sie germungen, tiefer berabudkommen. Das Liobt wirkt donn intensiver und erregt sie. Deshalb werden in truben, reguerischen oder nebligten Nächten die grölsten Züge konstatiert. Daher mulste sich solchen Beobachtern, die an ihrem Beobachtungsposten festgenagelt sind, die Ansicht aufdrängen, die Vogel sōgen in Mondnächten nicht, in hellen riesenhoch und kimen nur in donklen in die tieferen Schichten.

Dals Vogel anch beil Gewittern rieben, bat schon T. Hagerup nach Beobachtungen bei Kolding (Danemark) beschrieben (Orn. Monatsschrift 1905, p. 475-479). Die Vögel zieben selbst whihrend solcher Nachte, die mit geringen Unterbrechungen bis rum Morgen Gewitter neigen. In den Pansen sind die Züge besonders stark. Nie hörte ich die Vögel dann, wenn Bntladungen gerade tber dem Beobachter sind. Die Vögel scheinen den einzelDen Gewitterwolken ausumeichen. Naturlich rufen sie in Gewitternächten recht lebhsft.

\section{Ringexperimente.}

Wobl jeder wissenschaftlich tatige Feldornitbologe wird sich in den Dienst des Ringerperimentes stellen; sind doch durch dasselbe auf biologischem Gebiet Erfolge erzielt, die man am Anfang sicher nicht ahote. Auch ich habe die Sache ein wenig ru outerstituen versucht. Beringt sind: Störche, Sturmmöwen, Bibits, Austernfiscber, 1 Brandente, Amsel, Küsten- und Zrergseeschwalbe, Star, Saodregenpfeifer, Bucbfok, Grünfink, Goldammer, Heckenbraunelle, Sumpf- und Koblmeise, Gartenrotschwanz, Zaunkönig. Haus- und Ranchschwalbe, Haubenlerche.

Die Erfolge, die ich seit Sommer 1910 bis März 1912 errielte, möchte ich hier aufibren.

\section{Sturm $m$ to me (Larws cavus).}

Wahrend mit Lach- ond Silbermören sehr gute Resultate gewonnen sind, wurden mit Sturmmömen noch keine Versuche gemacht. Bs mulste mich daber reizen, solche mit diesen Vögeln Bnrustellen. Bekanut ist die Kolonie auf dem Langen Werder 
bei Poel. Es nisten dort ca. 7-800 Paare. Vor Anfang Juli kann ich jedoch nicht die Insel besuchen. Dann aber ist die grofse Mehrzahl der Jungen schon flügge und abgezogen. Beringt wurden im Laufe des Juli 191055 Stück, am 4. Juli 1911 46 Stück. Bis zum März 1912 wurden 10 zurückgemeldet. Davon wurden in Mecklenburg 3 gefunden, in Schleswig-Holstein 2, in Jütland 1, in Hannover 2, in Belgien und England je 1, sind $10 \mathrm{Ex}$.

1. Nr. 3756 , markiert am 12. Juli 10, am Brutplat nach eínigen Wch. tot gefunden vom Fischer Schwarz-Golwitz.

2. Nr. 3735, markiert am 12. Juli 10, wiedergef. wie Obige.

3. Nr. 4990, markiert am 4. Juli 11, am 22. August 11 bei Wilhelmshaven krank gegriffen.

Zeit: ca. 49 Tage.

Entfernung: $235 \mathrm{~km}$.

4. Nr. 4985, markiert am 4. Juli 11, am 13. Aug. 11 bei Harburg krank gegriffen.

Zeit: 40 Tage.

Entfernung: $117 \mathrm{~km}$.

5. Nir. 4970, markiert am 4. Juli 11, am 19. Aug. 11 bei Helgoland geschossen.

Zeit: 46 Tage.

Entfernung: $233 \mathrm{~km}$.

6. Nr. 7982, markiert am 4. Juli 11, in Schleswig (Mitte Okt.?) gefangen vom Fischer Reincke.

Zeit: ca. 100 Tage.

Entfernung: $134 \mathrm{~km}$.

7. Nr. 4981, markiert am 4. Juli 11, Mitte Nov. 11 in den Dünen von la Panne in Belgien (an der franz. Grenze) tot aufgefunden.

Zeit: ca. 135 Tage.

ca. $760 \mathrm{~km}$.

Entfernung: in der Luftlinie ca. $690 \mathrm{~km}$, am Strand

8. Nr. 4968, markiert am 4. Juli 11, am 20. Dez. 11 in Söhns, Möborg ved Boekmarksbro, bei Lemvig, West-Jütland, Dãnemark.

Zeit: 169 Tage.

Entfernung: $320 \mathrm{~km}$.

9. Nr. 4975, markiert am 4. Juli 11, am 3. Febr. 12 in Portsmouth (England) geschossen.

Zeit: 214 Tage.

Entfernung: $924 \mathrm{~km}$. (Nr. 4965 vom 4. Juli 1911 wurde im Okt. 11 am Brutplatz gefunden.)

Um wirklich exakte Schlüsse zu ziehen, mülste natürlich ein weit grölseres Material vorliegen. Aber einiges lälst sich klar erkennen.

1910 waren viele Jungmöwen eingegangen, anscheinend an Unterernährung, einige an Gelenkgeschwüren, vielleicht eine Folge von unverdaulichem Futter. Nur 2 sind, soeben flügge, 
am Brutort wiedergefunden. Nicht eine einzige wurde aus der Ferne zurückgemeldet. Dagegen sind von den 81911 wiedererlangten Möwen 6 anscheinend durch Krankheit auffindbar geworden, nur 2 sind geschossen. $1 \mathrm{~m}$ Sommer 1911 waren nur ganz wenig Jungmöwen eingegangen. Die Hitze war der Aufzucht anscheinend günstig. Dafür sind eine gröfsere Anzahl auf der Wanderung gefallen.

Einige wenige Sturmmöwen scheinen an der schleswigschen Küste hinaufzugehen. (Schleswig.) Die meisten folgen der „Lübecker Zugstrafse" nach SW (Harburg), gehen dann Elbe abwärts (Helgoland), folgen der friesischen Küste (Wilhelmshaven), der holländischen, belgischen (la Panue). Hier werden wahrscheinlich schon ihre Winterquartiere liegen, desgl. in England (Portsmouth). Bezeichnend ist jedenfalls, dafs von den 101 beringten Sturmmöwen im Winter nur eine von der östlichen Nordsee und keine von der Ostsee gemeldet ist, wo doch Sturmmöwen zahlreich überwintern, auch im Jugendkleide. Es scheint der Zug der Sturmmöwe ein Mittelding zwischen dem der Silbermöwe und der Lachmöwe $\mathrm{zu}$ sein. Jedenfalls fordern diese Erfolge dazu auf, weitere Markierungen zu unternehmen.

\section{He cke n b r a u e ll e (Prunella modularis).}

Mit Kleinvögeln habe ich in einer Gärtnerei bei Lübeck Versuche unternommen. Die Vögel werden hier $\mathrm{n}$ i c h t gefüttert. Der Ort ist uingeben von Laub- und Nadelwald, Feldern, Gärtnereien. Die Strafsen der Stadt grenzen daran. Die Gärtnerei liegt also nicht isoliert! Es ist also kein erkennbarer Grund zum besonderen Verweilen vorhanden.

Im Winter 1910/11 wurden 5 Braunellen beringt. Davon im selben Garten eine im stlben Winter wiedergefangen, eine zweite dort im folgenden Winter! Im Winter 1911/12 wurde keine gefangen, obgleich dort welche überwinterten.

1. Nr. 1428, markiert am 19. Januar 1911, wiedergefangen am 26. Januar 1911 und am 6. Februar 1911.

Zeit: 7 und 18 Tage.

2. Nr. 1432, markiert am 11. Februar 1911, wiedergefangen am 16. Januar 1912, 19. Januar 1912, am 2. Februar $1912\left(-28^{0}\right)$ tot aufgefunden. Schädeldach und rechte Bauchseite blutunterlaufen, Blutergufs ins Gehirn.

Zeit: 11 Monate 5 Tage, $11 \mathrm{Mt} .8 \mathrm{Tg}$, $11 \mathrm{Mt} .22 \mathrm{Tg}$. (nach 3 und 17 Tagen).

In den Hecken jener Gegend nistet die Braunelle nicht selten, überwintern tun dort regelmäfsig diese Vögel. Da kaum anzunehmen ist, dafs sie, aus nördlichen Gegenden kommend, $2 \mathrm{mal}$ am selben Ort überwinterten, ist es wahrscheinlich, dafs diese überwinternden Vögel Brutvögel der Gegend sind. 


\section{K o h l me i se (Parus maior).}

Es sind im Winter 1910/11 5 Stück beringt, davon ist nur eine nicht wieder gefangen. Im Winter 1911/12 sind 3 markiert, alle wieder gefangen. Leider ist keins vom vorigen Winter zurückerlangt. Es ist nach der Zeit geordnet:

1. Nr. 1429, markiert am 19. Januar 1911, gefangen am 22. Januar 1911.

\section{Zeit: 3 Tage.}

30. März 1911.

2. Nr. 1427, markiert am 20. Januar 1911, gefangen am

Zeit: 69 Tage (2 Mt. 10 Tg.).

3. Nr. 1426, markiert am 21. Januar 1911, gefangen am 3. Febr. und 2. Mai 1911.

Zeit: 13 und 101 Tage (4 Mt. 11 Tg.).

4. Nr. 1431, markiert am 14. Okt. 1910, gefangen am 8. Jan., 17. Jan., 24. Jan., 7. Febr. und 21. April 1911.

Zeit: 86 Tg., 95 Tg., 102 Tg., 116 Tg. und 189 Tg. (6 Mt. 7 Tg.).

Gesehen sind während des Winters oft beringte Kohlmeisen in der Hofstelle. Sie bielten sich nicht ausschliefslich stets darin auf, sondern kehrten öfters vor, Die Meisen im April und Mai sind im Nistkasten gefangen, ein Zeichen, dafs sie dort brüten wollten. Tatsächlich hat in einem hohlen Apfelbaum, wo alljährlich ein Paar nistet, ein Pärchen Junge grofs gezogen, bei dem beide Vögel Ringe trugen. Als ich das Paar fangen wollte, waren die Jungen ausgeflogen. Es bleiben also unsere Brutvögel hier und haben den Wandertrieb gänzlich verloren, und zwar Männchen und Weibchen. Ende Mai 1912 brütete wieder eine Ringmeise im Garten.

5. Nr. 2869, markiert am 5. Nov. 1911, gefangen am 5. Dez. und 10. Dez. 1911.

Zeit: 30 und 35 Tage ( 1 Mt. 5 Tg.).

6. Nr. 2870, markiert am 5. Nov. 1911, gefangen am 28. Januar 1912.

\section{Zeit: 84 Tage (2 Mt. 13 Tg.).}

7. Nr. 2871, markiert am 30. Nov. 1911, tot aufgefunden am 8. März 1912, vielleicht einige Tage alt, da schon etwas riechend.

Zeit: 99 Tage (3 Mt. 7 Tg.).

IV. S u m p m e is e (Parus palustris).

Von 2 Stück eine wieder.

Nr. 2868, markiert am 4. Nov. 1911, am 7. Nov. 1911 von Katze getötet.

Zeit: 3 Tage. 


\section{A m sel (Turdus merula).}

Im Winter 1910/11 wurden 2 Stück beringt, davon 1 zurück.

1. Nr. 1723 우, im Januar 1911 gefangen, in grofser Gartenvolière gehalten, am 9. April 1911 in Freiheit gesetzt. Am 25. Januar 1912 im selben Garten im Kanincheneisen geschlagen.

Zeit: 291 Tage.

Im Winter 1911/12 sind 12 Stück markiert, davon 4 zurück.

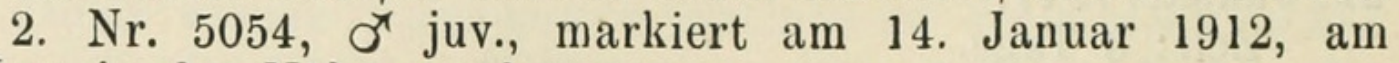
28. Jan. in der Nähe geschossen.

Zeit: 14 Tage.

Trotzdem im Januar grofse Kälte $\left(-15^{\circ}\right)$ einsetzte, ist dieser junge Vogel nicht abgezogen.

3. Nr. 5056, $\sigma^{\Upsilon}$ ad., markiert am 28. Januar, Mitte Febr. tot gefunden.

4. Nr. 1710 (Schw.-R.), ơ ad., markiert am 27. Januar, Mitte Febr. im Nachbargarten tot gefunden.

5. Nr. 5058, $\sigma^{x}$ ad., in den ersten Tagen des Februars markiert, Mitte Febr. tot gefunden.

Die grofse Kälte $\left(-28^{\circ}\right)$, die in der 2. Februarwoche herrschte, hat viele Vögel getötet. Auch diese Drosseln sind ihr zum Opfer gefallen.

\section{R a u c h s ch w a l be (Hirundo rustica).}

Im Sommer 1910 wurden die Alten und flüggen Jungen eines Nestes bei Lübeck markiert. Im April 1911 erschien in der Scheune (wo nur 1 Paar stets nistete) ein Pärchen wieder. Wie öfters festgestellt wurde, auch mittels Glas, hatten beide Vögel Ringe. Ich wollte die Vögel nicht stören, da Schwalben dafür sehr empfindlich sind, und wollte bis zur Brutvollendung mit dem Fang warten. Nach einigen Wochen, als die Vögel schon öfters auf dem Neste safsen, also brüten wollten, wurden die Flügel des einen Stückes gefunden. Es war von der Katze geschlagen. Der andere Vogel verschwand. Nach mehreren Wochen kamen wieder 2 am Nest. Davon hatte der eine einen Ring, der 2. nicht. Die Vögel safsen öfters auf dem Nest, brüteten jedoch nicht. Da nun Dr. Thienemann Schwalben in der Nähe ihres Nestes wiedergefangen hat, unterliegt es für mich keinem Zweifel, dafs dieses Pärchen das Brutpaar vom vorigen $\mathrm{Jahr}$ ist.

Wie Prof. Möbius (Das Wandern der Deutschen Sommervögel, Himmel und Erde, XII. J., H. 1, p. 6) nach The Zoologist 3. Ser. XIX, 1895, p. 449, mitteilt, wurden in England am 6. Juni 1893 zwei Hausschwalben mit Fufsringen markiert. Sie kamen am 20. Juni 1894 wieder an der Niststelle an. 

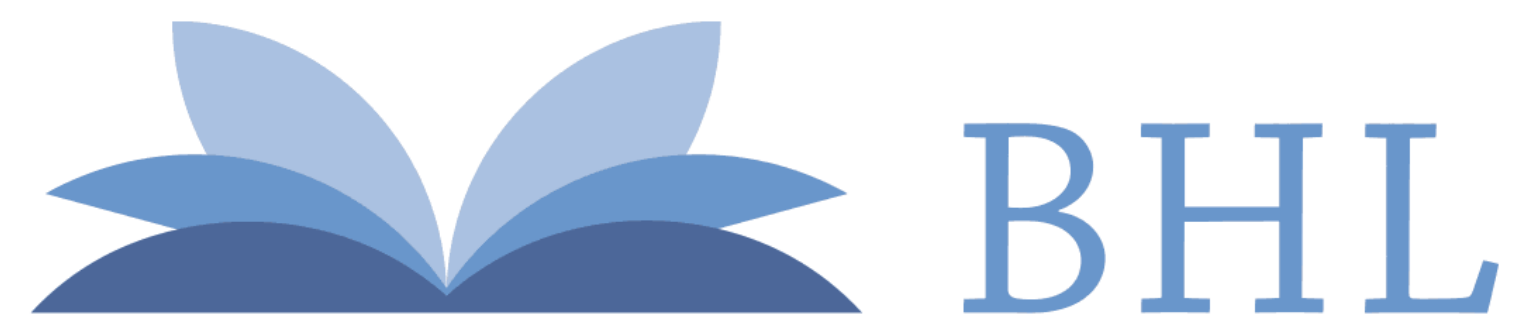

\section{Biodiversity Heritage Library}

1912. "Über den Vogelzug 1911 bei Lübeck." Journal

fu

r Ornithologie 60, 470-480. https://doi.org/10.1007/bf02090184.

View This Item Online: https://www.biodiversitylibrary.org/item/43502

DOI: https://doi.org/10.1007/bf02090184

Permalink: https://www.biodiversitylibrary.org/partpdf/142739

\section{Holding Institution}

MBLWHOI Library

\section{Sponsored by}

MBLWHOI Library

\section{Copyright \& Reuse}

Copyright Status: No known copyright restrictions as determined by scanning institution.

This document was created from content at the Biodiversity Heritage Library, the world's largest open access digital library for biodiversity literature and archives. Visit BHL at https://www.biodiversitylibrary.org. 